\title{
Evolving invasive neonatal systemic candidiasis, a review
}

\begin{abstract}
Preterm and low birth weight (LBW) infants are vulnerable to invasive candidiasis, usually caused by Candida albicans and Candida parapsilosis. Neonatal candidiasis, acquired after 6 days of life, is the most common form of invasive candidiasis in neonates. Once the Candida species penetrate any organ system, it can lead to complications such as meningitis, endocarditis, pyelonephritis, septic arthritis and pneumonia. In recent years, the incidence of invasive candidiasis has decreased in neonatal intensive care units (NICU) due to improvements in neonatal care; however, in the affected neonates, mortality remains a major concern. This is primarily attributable to the limitations of currently used diagnostic tests and delay in treatment. In addition, there is a lack of data on pharmacokinetics and pharmacodynamics of various anti-fungal therapeutic regimens in neonates. This review provides an overview of invasive candidiasis in neonates, including modes of transmission, its risk factors, and management, with special focus on the recent updates in diagnosis and treatment.
\end{abstract}

Keywords: anti-fungal, invasive candidiasis, neonatal candidiasis, neonates, systemic candidiasis, management
Volume 6 Issue 6 - 2017

\author{
Khaled El-Atawi,' Mahmoud Elhalik, ${ }^{2}$ \\ Tushar Kulkarni, ${ }^{3}$ Amany Abdelsamed, ${ }^{4}$ Lois \\ Alexander, ${ }^{4}$ Aswathy Devaki Satyan, ${ }^{4}$ Rim \\ Mahfouz $^{4}$ \\ 'Consultant Neonatologist, Neonatal Intensive Care Unit, \\ Pediatric Department, Latifa Women \& Children Hospital, Dubai \\ Health Authority, UAE \\ ${ }^{2}$ Consultant Neonatologist and Head of Pediatric Department \\ \& Neonatal Intensive Care Unit, Latifa Women \& Children \\ Hospital, Dubai Health Authority, UAE \\ ${ }^{3}$ Specialist Senior Registrar, Neonatal Intensive Care Unit, \\ Pediatric Department, Latifa Women \& Children Hospital, Dubai \\ Health Authority, UAE \\ ${ }^{4}$ Specialist Registrar, Neonatal Intensive Care Unit, Pediatric \\ Department, Latifa Women \& Children Hospital, Dubai Health \\ Authority, UAE
}

\author{
Correspondence: Khaled El-Atawi, NICU, Pediatric \\ Department, Latifa Women \& Children Hospital, Dubai, UAE, \\ Email kelatawi@eim.ae
}

Received: April 27, 2017 | Published: June 30, 2017

\section{Introduction}

Preterm and low birth weight (LBW) infants are commonly susceptible to invasive candidiasis, usually caused by Candida albicans and Candida parapsilosis. ${ }^{1-5}$ Other nonalbicans Candida species found to be associated with invasive candidiasis include: C. glabrata, C. tropicalis, C. lusitaniae, C. guilliermondii, C. pelliculosa, C. zeylanoides and C. krusei . ${ }^{1-4}$

Invasive candidiasis can either be acquired in-utero (within 6 days of life) or post-partum (after 6 days of life). If acquired in-utero, it is referred as congenital candidiasis (CC). CC is usually cutaneous initially and becomes systemic later, known as congenital systemic candidiasis. It is a rare form of invasive candidiasis with very few cases (around 100) reported in medical literature. ${ }^{6,7}$ The other type of infection is acquired after 6 days of life and is referred as neonatal candidiasis. It is the common form of invasive candidiasis in neonates.

Once the Candida species enter blood stream, it is likely to penetrate in various body organs including central nervous system (CNS), heart, kidneys, liver, spleen and eyes. This can lead to complications such as meningitis, endocarditis, candida pyelonephritis, renal papillary necrosis, multiple parenchymal abscesses, endophthalmitis, septic arthritis and osteomyelitis, peritonitis, and pneumonia. ${ }^{8-10}$

With advances in neonatal management, incidence of invasive candidiasis has decreased in neonatal intensive care units (NICUs) over the years. A study by Aliaga et al. (2014) reported a decrease in the annual incidence of invasive candidiasis from 3.6 episodes per 1000 patients to 1.4 episodes per 1000 patients among all infants from 1997 to 2010 . On the basis of birth weight, the incidence decreased from 24.2 to 11.6 episodes per 1000 patients among infants with a birth weight of $750-999 \mathrm{~g}$, and from 82.7 to 23.8 episodes per 1000 patients among infants with a birth weight $<750$ g. ${ }^{11}$ Another study by
Chitnis et al. (2010) assessed the incidence of Candida species central line-associated bloodstream infections (CLABSIs) in US NICUs from 1999 to 2009 . The study results showed a significant decrease in the incidence of CLABSIs due to Candida species (pooled mean rate of Candida spp. CLABSIs per 1000 central line-days reduced from 0.9 in 1999 to 0.2 in $2009 ; p<0.001) .{ }^{12}$ However, the mortality rate associated with invasive candidiasis is reported to range from $19.6 \%$ to $63 \%$ and remains a major concern. ${ }^{1,4,13}$

Despite the decrease in incidence of invasive candidiasis in neonates, the associated mortality rate is high in preterm and very LBW infants. High mortality can be attributed to the limitations of routinely used diagnostic tests (such as longer duration and insufficient amount of sample for testing) and delay in treatment (due to longer time taken for confirmation of diagnosis and lack of sufficient data on dosing of antifungal therapy in neonates). ${ }^{8,10,14}$ Although, various antifungals have been developed for treatment of invasive candidiasis, there is scarcity of data on their pharmacodynamics and pharmacokinetics in neonates and children. ${ }^{15}$ All these factors highlight the need for appropriate diagnosis and management of neonatal candidiasis.

The present review discusses the overview of invasive candidiasis in neonates, modes of transmission, its risk factors, and neurodevelopmental outcomes. Further, the review explains the diagnosis and treatment of invasive candidiasis in neonates, with special focus on the recent updates in its management.

\section{Modes of transmission}

Candidalinfections can be transmitted in neonates via two modes:

i. Vertical transmission: It occurs due to maternal vaginal infection. C. albicans is responsible for $90 \%$ of the vaginal fungal colonization during pregnancy. Increased adherence 
of Candida to intermediate layers in the vaginal tract increases the risk of exposure of infants delivered vaginally to candidal infections. ${ }^{10} \mathrm{CC}$ occurs via vertical transmission and has been reported to occur rarely in medical literature. ${ }^{6}$

ii. Horizontal transmission: It occurs nosocomially i.e. acquired in hospitals, and is transmitted via contaminated medical devices, hands of healthcare workers, catheters, etc. C. parapsilosis is found to be the most common Candida species present on the hands of healthcare workers. ${ }^{16}$

\section{Risk factors}

Multiple risk factors in neonates contribute to invasive candidiasis LBW (>2500 g) and earlier gestational age (29-32 weeks) are found to be commonly associated with invasive candidiasis in neonates. Other risk factors identified in clinical studies to be associated with invasive candidiasis are vaginal birth, central catheters, day of life $(>7)$, use of broad-spectrum antibiotics in neonates, antenatal steroids, premature rupture of membranes, mechanical ventilation, necrotizing enterocolitis and parenteral nutrition (Table 1).,14,13,17

\section{Neurodevelopment outcomes in neonates with invasive candidiasis}

Invasive candidiasis in neonatal survivors is found to be associated with adverse neurodevelopmental outcomes. A study by Benjamin et al. (2006) reported neurodevelopmental impairment (NDI) or mortality in $73 \%$ of infants with invasive candidiasis. Moreover, infants who had delayed removal or replacement of central catheters ( $>1$ day after initiation of antifungal therapy) were at increased risk of NDI. ${ }^{18}$

In a study by De Hann et al.(2013), 29 neonates who survived Candida sepsis were followed up at 24 months to assess the neurological outcomes. Of the 29 neonates, 17 appeared for follow-up, and neurological examination showed mild and severe abnormality in $9(53 \%)$ and $1(6 \%)$ infants, respectively. The median value of Bayley Psychomotor Development Index (PDI) and Bayley Mental Development Index (MDI) were 76 (59-100) and 92 (78-108), respectively. Hearing disabilities and visual impairment occurred in $4(24 \%)$ and $3(18 \%)$ infants, respectively. ${ }^{19}$ Another study by Adams-Chapman et al. (2013) assessed the neurodevelopmental outcomes of extremely LBW infants who survived following sepsis with Candida infection or non-Candida sepsis at 18 months of age. The study reported that $31 \%$ of infants in each group (with Candida sepsis and non-Candida sepsis) had NDI at 18 months. However, infants with Candida sepsis (OR: 1.83; 95\% CI: $=1.83$ [1.01-3.33], $p=0.047$ ) were reported to be at increased risk of NDI as compared to uninfected infants. ${ }^{20}$

Another study assessed the neurodevelopmental outcomes in infants $(\leq 1000 \mathrm{~g})$ with invasive candidiasis who were receiving empirical antifungal therapy. The study reported that incidence of death or NDI was less in infants who received empirical antifungal therapy $(19 / 38,50 \%)$ compared with those who had not $(55 / 86$, $64 \%$; OR $=0.27$ [95\% CI 0.08-0.86]. It was suggested that empirical antifungal prophylaxis should be initiated in LBW infants with risk of fungal infections to improve neurodevelopmental outcomes. ${ }^{2}$

\section{Management of invasive candidiasis}

\section{Diagnosis}

Rapid diagnosis is the best possible way to successfully manage the preterm or full-term neonate with invasive candidiasis.
Body fluids culture: Blood culture is the most common diagnostic procedure used for detection of candidiasis. ${ }^{14}$ Other commonly used methods include culture/microscopic examination of urine, cerebrospinal fluid, peritoneal fluid and other sterile body fluids. ${ }^{10}$

However, blood culture to confirm diagnosis of invasive candidiasis in neonates has several limitations. Firstly, the availability of less amount of blood ( 0.5 to $1 \mathrm{~mL}$ ) for culturing makes it difficult to isolate Candida in neonates. Secondly, the median time taken by blood culture to detect Candida species in infants is $36 \mathrm{hrs}$ and might reach $42 \mathrm{hrs}$, if the infant is receiving antifungal therapy prior to blood culturing. ${ }^{14}$ Further, speciation and susceptibility testing following isolation from blood culture adds on more days for diagnosis. ${ }^{21}$

Also, the negative cerebrospinal fluid (CSF) culture in infants does not exclude the presence of candida meningitis. A study reported presence of normal CSF parameters in almost half of the study patients with Candida meningitis. ${ }^{22}$. Thus, new diagnostic methods are being investigated to overcome the limitations associated with the culturing method.

\section{Rapid diagnostic methods}

Peptide nucleic acid fluorescence in situ hybridization (PNA FISH): Once the blood culture turns out to be positive, technique like PNA FISH can be used to shorten the time required for speciation. ${ }^{21}$ A recent study compared the compatibility of the peptide nucleic acid fluorescent in situ hybridization Yeast Traffic Light (PNA FISH ${ }^{\circledR}$ YTL) with VITEK 2 System in early identification of Candida spp. directly from positive blood cultures $(n=15)$ and other clinically significant specimens $(\mathrm{n}=10)$. The study reported assay identification compatibility of PNA FISH ${ }^{\circledR}$ YTL with VITEK 2 System in 21/25 ( $84 \%$ ) specimens tested, and suggested that this assay can be used to identify Candida from blood cultures, urine cultures, peritoneal fluid cultures and catheter tip cultures. ${ }^{23}$ Although this test appears to be clinically beneficial, its use is limited due to several factors such as lack of specificity, requirement of a positive blood culture, and inability to distinguish between all clinically relevant Candida species. ${ }^{21}$

Polymerase chain reaction: Is being studied to identify Candida infection in neonates more rapidly and with higher sensitivity. PCR also makes it feasible to use small amount of blood to detect Candida infection. ${ }^{10,24,25}$ Table 2 presents studies evaluating the use of PCR in neonates with invasive candidiasis.

A meta-analysis of 23 studies evaluating efficacy of PCR and other hybridization methods in diagnosis of neonatal sepsis concluded that molecular techniques cannot replace culture methods presently due to insufficient sensitivity, although they can be used as an add-on to culture methods..$^{26}$ A review by Paolucci et al. highlighted that lack of sensitivity could be attributed to varied amount of blood collected in different studies ( $200 \mu \mathrm{L}$ to $2 \mathrm{~mL}){ }^{27}$ Moreover, there is lack of studies on standardization and validation of PCR in neonates. ${ }^{28}$ PCR use is further limited due to likelihood for sample contamination (leading to false-positive results), lack of probes with capability to detect multiple Candida species, and difficulty in sample preparation. ${ }^{21}$ Thus, more studies need to be conducted to assess the validity and reliability of PCR and other molecular techniques in neonates.

Biomarkers: Researchers are also investigating the use of markers of fungal disease in diagnosis such as $(1,3)-\beta$-D-Glucan (present in fungal cell wall), D-arabinitol, anti-Candida antibodies, mannan antigen, and fungal chitin synthase. ${ }^{10,29}$ Very few studies have been conducted in neonates on usability of these biomarkers in diagnosis of invasive candidiasis (Table 2). Although these tests show encouraging 
results, cost and lack of substantial data in neonates prevent their use in standard practice presently.

Other diagnostic tests: Neonates with persistent candidemia are likely to have end-organ dissemination (EOD). Thus, it is suggested to screen EOD using renal and cranial ultrasound/ magnetic resonance imaging (MRI), echocardiogram and ophthalmologic examination, either prior to initiation of the therapy or after 5-7 days of the initiation of the treatment. Abdominal ultrasound is suggested to assess for peritoneal, splenic or liver involvement. ${ }^{10}$

Table I Studies Assessing Risk Factors for Invasive Candidiasis in Neonates.

\begin{tabular}{|c|c|c|}
\hline & Patient population & Risk factors \\
\hline \multirow[t]{2}{*}{ Barton et al. ${ }^{13}$} & $N$ (cases) $=49$ & NEC (OR: $\left.4.81 \quad .95 \% \mathrm{Cl}: !^{14-20.41} ; p=0.03\right)$ \\
\hline & & $\begin{array}{l}\text { Day of life }>7(\text { OR } 25.2 ; 95 \% \text { Cl I4.6-43.3) } \\
\left.\text { Vaginal birth (OR I.6 } \text {.. }^{2-2.3} .^{3}\right)\end{array}$ \\
\hline Lee et al.. ${ }^{51}$ & $N=330(>1500 \mathrm{~g})$ & 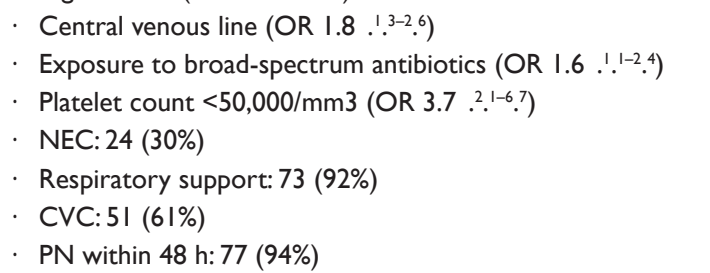 \\
\hline Oeser et al..$^{52}$ & $N=98$ & $\begin{array}{l}\text { Trophic feeds within } 48 \mathrm{~h}: 52 \text { (63\%) } \\
\text {. } \\
\text { Receipt of systemic corticosteroids: } 8 \text { (I0\%) } \\
\text { H2 receptor antagonists: } 10 \text { (I2\%) } \\
\text {. Insulin } 16 \text { (20\%) } \\
\text { Previous abdominal surgery: II (I3\%) } \\
\text {. Intrapartum use of broad-spectrum antibiotics: } 59 \text { (95.16\%) } \\
\text {. Prematurity: } 38(61.29 \%) \\
\text {. LBW: } 47(79.03 \%)\end{array}$ \\
\hline Wadile et al..$^{53}$ & $N=108$ & $\begin{array}{l}\text { Indwelling catheters: } 5(8.06 \%) \\
\text { Artificial ventilation: } 26(41.94 \%) \\
\text { Male sex: } 37(59.67 \%) \\
\text {. Vaginal delivery: } 53(85.48 \%)\end{array}$ \\
\hline Khan et al. ${ }^{17}$ & $\begin{array}{l}N=560(36 \%) \text { had positive } \\
\text { cultures. Candida was isolated in } 49 \\
(8.8 \%) \text { neonates with positive cultures. }\end{array}$ & $\begin{array}{l}\text { Mechanical ventilation: } 4 \text { I }(83.7 \%) \\
\text { Prior antibiotic therapy: } 45(92 \%) \\
\text {. Partial PN I5 (30\%) }\end{array}$ \\
\hline
\end{tabular}

*NEC, necrotizing enterocolitis; PN, parenteral nutrition

Table 2 Studies Assessing the Use of Molecular Diagnostic Tests in Neonatal Candidiasis.

\begin{tabular}{|c|c|c|c|c|}
\hline Studies & Type of Study & Patient population & $\begin{array}{l}\text { Diagnostic } \\
\text { technique assessed }\end{array}$ & Outcomes \\
\hline Zhao et al. ${ }^{29}$ & Case control study & $\begin{array}{l}63 \text { preterm infants with IFD, } 160 \\
\text { preterm infants without sepsis } \\
\text { (preterm control), and } 41 \text { preterm } \\
\text { infants with bacterial sepsis }\end{array}$ & $\begin{array}{l}\text { BG and platelet } \\
\text { parameters (PC, PCT, } \\
\text { PDW and MPV) }\end{array}$ & $\begin{array}{l}\text { Sensitivity and specificity of BG: } 68.3 \% \\
\text { and } 75.6 \% \text {; PC: } 78 \% \text { and } 95 \% \text {, and PCT: } \\
83 \% \text { and } 85 \% \text {, respectively }\end{array}$ \\
\hline Taira et al..$^{25}$ & Prospective study & 54 pediatric patients ( 24 neonates) & $B C$ and $P C R$ & $\begin{array}{l}\mathrm{BC} \text { and multiplex nested PCR were } \\
\text { positive in } 14.8 \% \text { vs } 24.0 \% \text { of patients, } \\
\text { respectively. }\end{array}$ \\
\hline Goudjil et al. ${ }^{54}$ & Retrospective study & $\begin{array}{l}\text { Infected }(n=18) \text { or non-infected }(n \\
=43) \text { neonates }\end{array}$ & BG I & $\begin{array}{l}\text { BG level was significantly higher in the } \\
\text { infected group }\left(364 \mathrm{pg} / \mathrm{mL} \cdot{ }^{13-1976}\right) \text { as } \\
\text { compared to non-infected group ( } 89 \\
\left.\mathrm{pg} / \mathrm{mL} \cdot .^{30-127} ; \mathrm{p}<0.00 \mathrm{I}\right) .\end{array}$ \\
\hline Oliveri et al..$^{55}$ & Observational study & $\mathrm{N}=70$ pre-term infants & Mannan antigen test & $\begin{array}{l}\text { Overall sensitivity and specificity } \\
\text { of the assay was } 94.4 \% \text { and } 94.2 \% \text {, } \\
\text { respectively and culture-proven } \\
\text { sensitivity and specificity was } 92 \% \text { and } \\
84 \% \text {, respectively }\end{array}$ \\
\hline Mularoni et al. & Case series & $\begin{array}{l}\mathrm{N}=4 \text { ( } 2 \text { low-birth-weight } \\
\text { neonates and } 2 \text { stem cell transplant } \\
\text { recipients) }\end{array}$ & BG test & $\begin{array}{l}\text { Plasmatic levels of BG were }>523 \mathrm{pg} / \\
\mathrm{mL} . \mathrm{BG} \text { test might be used to identify } \\
\text { IFD in pediatrics }\end{array}$ \\
\hline
\end{tabular}

*IFD, invasive fungal disease; BG, (I, 3)- $\beta$-D-glucan; PC: Platelet count, PCT plateletcrit; PDW, platelet distribution width; MPV, mean platelet volume; BC, blood culture 


\section{Treatment}

There is lack of data on efficacy and safety of antifungals in neonates. The studies conducted till date have only focused on pharmacokinetics and pharmacodynamics evaluations of the antifungal agents. However, the recent updated guidelines from the Infectious Diseases Society of America (IDSA) (2016) presented recommendations for management of invasive candidiasis in neonates based on the available clinical data. The European Society of Clinical Microbiology and Infectious Disease (ESCMID) also released guidelines on the diagnosis and management of invasive candidiasis in neonates. ${ }^{30}$

\section{Guideline recommendations and optimal management}

1. Pharmacological treatment: Figure 1 presents an overview of recommendations by IDSA on antifungal therapy in neonates with special recommendations for neonates with CNS involvement and those in ICU. ${ }^{31}$

i. Amphotericin: IDSA guidelines recommends Amp B deoxycholate (D-Amp B) as the first treatment option for neonates with disseminated candidiasis (category A-II). ${ }^{31}$ ESCMID guidelines also recommend the use of D-Amp B in neonates with invasive candidiasis, but the recommendation is supported by limited clinical data as well as pharmacokinetic data (category B-II). ${ }^{30} \mathrm{D}$-Amp B is reported to be associated with nephrotoxicity. Holler et al. conducted a study to assess the effects of fluid and electrolyte management on reducing the Amp B-induced nephrotoxicity among 753 extremely LBW infants with systemic fungal sepsis. The study showed that Amp B when given with adequate hydration and increased sodium intake $(>4 \mathrm{mEq} / \mathrm{kg} /$ day) may help in reducing the associated nephrotoxicity. ${ }^{32}$

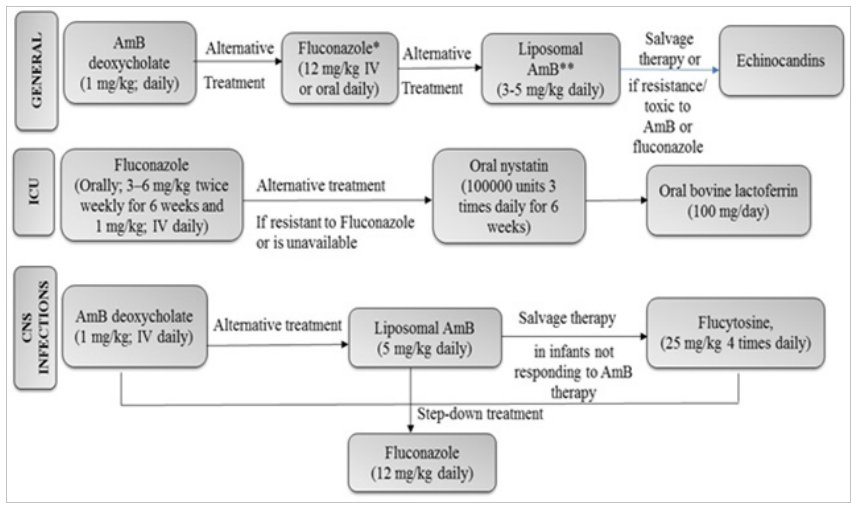

Figure I Summary of Recommendations on Management of Invasive Candidiasis in Neonates as per IDSA Guidelines.

IV: intravenous; CSF, cerebrospinal fluid; CNS, central nervous system; ICU,Intensive care unit, Amp B, amphotericin B.

*In patients who have not been on fluconazole prophylaxis

**To be used cautiously, particularly in the presence of urinary tract involvement. ${ }^{50}$

The lipid-based formulations i.e. amphotericin B liposomal complex (ABLC) are also being used to treat invasive candidiasis in neonates. ABLC has received a grading of B-III and B-II in ISDA and ESCMID guidelines, respectively, for use in neonates with invasive candidiasis. ${ }^{30,31}$ Although the safety of liposomal formulations of Amp
B has only been assessed in some observational uncontrolled studies, results have been favorable. ${ }^{33,34}$ However, there is lack of recent data evaluating the safety of ABLC in neonates. Moreover, there are few studies comparing ABLC with other therapies including Amp B. A recent study compared the use of ABLC with conventional Amp B in infants ( $\leq 120$ days of age) who had confirmed diagnosis of invasive candidiasis. The study reported increased mortality rate and treatment failure for neonates receiving lipid formulations compared with Amp (OR: 1.96 [95\% CI: 1.16, 3.33]; $\mathrm{p}=0.01 .^{35}$ Further well-designed studies (randomized) need to be conducted to assess the efficacy and safety of ABLC in neonates.

ii. Fluconazole: Fluconazole is recommended as an alternative treatment option for neonates with candidemia and invasive candidiasis and as the first treatment option for neonates in intensive care units. ${ }^{31}$ However, results of the recent study by Benjamin et al. (2014) showed that fluconazole prophylaxis (for 42 days) in infants with a birth weight $<750 \mathrm{~g}$ did not show reduction in the incidence of composite of death or invasive candidiasis. ${ }^{36}$ Another recent study by Lee et al. (2016) also assessed efficacy and safety of fluconazole prophylaxis in comparison to non-prophylaxis in infants with extremely LBW in NICU. The study reported that fungal colonization rate reduced significantly with fluconazole prophylaxis (59.1 $\%$ vs. $33.9 \%, \mathrm{p}<0.001)$. However, fluconazole prophylaxis resulted in increased incidence of invasive infections involving fluconazole-resistant $C$. parapsilosis ( $0 \%$ vs. $41.7 \%, p=0.11$ ), although it was statistically insignificant. This study also supports the findings that fluconazole is not efficacious in infants with extremely LBW in reducing invasive fungal infections. ${ }^{37}$ Also, there is a need for clinical studies to be conducted to assess the long-term effect of fluconazole therapy on development of antifungal resistance. ESCMID guidelines recommend that the decision to use fluconazole prophylaxis in NICUs with lower incidence of invasive candidiasis $(<2 \%)$ should be made on case-by-case basis. ${ }^{30}$

iii. Micafungin: Echinocandin is also considered to be a first lineagent based on clinical data in neonates. ${ }^{38} \mathrm{~A}$ study by Arrieta et al. reported that micafungin is safe in children of all ages. This study included a total of 296 patients $(6.5 \pm 5.1$ years), of which 66 were $<1$ year of age and 38 were premature (received $\geq 1$ dose of micafungin). ${ }^{39}$ Another study by Benjamin et al. also showed similar results supporting safety of micafungin in young infants. ${ }^{40}$ However, ESCMID guidelines state that 'black box' warning has been issued by the European Medical Agency (EMA) indicating the use of micafungin only if other agents are not appropriate. EMA has issued this warning due to an increased incidence of hepatic tumors in pre-clinical studies with the use of micafungin for longer durations. ${ }^{30} \mathrm{~A}$ recent systematic review of 9 clinical trials assessing the safety and efficacy of micafungin in premature and non-premature infants with invasive candidiasis showed the drug to be well tolerated, but was discontinued due to treatment-related adverse events (TEAEs) in some patients. A total of $2(4 \%)$ premature and $7(12 \%)$ non-premature patients $(p=0.17)$ discontinued the treatment following TEAEs. ${ }^{41}$

iv. Caspofungin: Caspofungin, an echinocandin, was compared to $\mathrm{AmpB}$ in neonates with invasive candidiasis to assess its efficacy and safety. Thirty-two (32) neonates with invasive candidiasis were randomized to receive either caspofungin $(n=15)$ or AmpB $(n=17)$. Caspofungin showed efficacy in 
$86.7 \%$ of patients as compared with $41.7 \%$ of those who received AmpB $(p=0.04)$. Adverse events were significantly lower in the caspofungin group than in the AmpB group. ${ }^{42}$ Jeon et al. (2014) also reported the efficacy and safety of Caspofungin $(2 \mathrm{mg} / \mathrm{kg} /$ day) in treating premature infants $(\mathrm{n}=$ 7; gestational age: $23+1-24+6$ weeks, and birth weight: 530 $825 \mathrm{~g})$ with persistent candidemia. Caspofungin successfully treated persistent candidemia in all the infants and no adverse effects were reported. None of the infants was found to have recurrent candidemia after discontinuing caspofungin. ${ }^{43}$ Although various studies have shown capsofungin to be safe and effective in invasive candidiasis in neonates, the population size in these studies was less. More studies with large population size needs to be conducted. Presently, a grading of C-II is given for capsofungin in ESCMID guidelines. ${ }^{30}$

v. Combination therapy: There is a lack of substantial data on the use of combination therapy in neonates with invasive candidiasis. Conflicting results have been obtained in different studies. A recent case report by Al-Sweih et al. (2015) reported successful treatment of persistent candidemia in a preterm neonate caused by Candidafermentati with combination therapy of AmpB and caspofungin (for 30 days). No adverse effects were reported to occur in the patient. ${ }^{44}$ Another study reported the use of liposomal AmpB and voriconazole in 6 infants with very LBW. These newborns were initially treated with AmpB (either conventional or liposomal) and fluconazole. However, 10 infants developed Candida sepsis and candidemia persisted in 6 infants despite receiving these antifungals for 3-21 days. Later, voriconazole was added as a combination therapy to AmpB which resulted in Candida clearance in 3-7 days. Thus, the study results showed that Voricazole can be used in combination with Amp B for treatment of fungal sepsis in neonates who still have persistent candidemia despite conventional antifungal management. ${ }^{45}$

On the other hand, a study by Ascher et al. reported the use of D-Amp B, fluconazole, Amp B lipid products, or combination therapy in 730 infants. Multivariable regression showed no difference in the duration of candidiasis for infants treated with D-Amp B, Amp B lipid products, fluconazole, or combination therapy $(p=0.47)$. There was no significant difference in therapy failure rates for fluconazole and combination therapy compared with D-Amp B. ${ }^{35}$ No recommendation has been made in guidelines on the use of combination therapy in neonates. $^{31}$

Other antifungals: Use of some other antifungals including Itraconazole, Voriconazole and anidulafungin have been assessed in very few studies in neonates, mainly limited to case reports. Thus, their use is not recommended in clinical practice ${ }^{46}$ Several new antifungals such as Posaconazole, Ravuconazole, 5-Flucytosine, Isavuconazole and Posaconazole have also not been studied in neonates so far. ${ }^{38,46}$

Probiotics: Several studies are being conducted to assess the use of probiotics in treatment of late onset sepsis (LOS) in neonates. A recent meta-analysis by Rao et al. systematically reviewed 37 randomized clinical trials comparing probiotics with placebo and control in preterm infants with LOS. Probiotics significantly decreased the risk of LOS (675/4852 [13.9\%] vs 744/4564 [16.3\%]; relative risk [RR]: 0.86; $95 \%$ CI: $0.78-0.94 ; \mathrm{p}=0.0007) .{ }^{47} \mathrm{~A}$ meta-analysis by Zhang et al. (2016) also reported significant reduction in fungal sepsis (RR $0.57,95 \% \mathrm{CI}$ : $0.41-0.78$ ) with enteral probiotic supplementation in preterm neonates with LOS ${ }^{48}$ Although several studies have shown that probiotics play an important role in preventing Candida colonization in neonates with invasive candidiasis (IC), more multicenter clinical trials are needed to evaluate efficacy, safety, dosage, duration and strains (type and single or combination of different strains) of probiotics in prevention of Candida colonization and invasive candidiasis. ${ }^{49}$ Lactoferrin alone or in combination with Lactobacillus is suggested to be equally useful by the ESCMID guidelines. ${ }^{30}$. However, no recommendation on their use is given in the recent updated guidelines by ISDA.

An expert opinion states that researchers need to conduct studies assessing the efficacy and safety of antifungals in neonatal population because data from adult trials cannot be implied to neonates with invasive candidiasis due to differences in pathophysiology of the disease in neonates and adults. ${ }^{46}$

2. Non-Pharmacologic treatment: The guidelines from IDSA strongly recommend the removal of central venous catheter (CVC) in neonates as a part of non-pharmacologic treatment. ${ }^{31}$ In a study by Benjamin et al. (2006), delayed catheter removal was significantly associated with higher mortality rate $(37 \% v s$. $21 \% ; p<0.024)$ in extremely LBW infants with invasive candidiasis. ${ }^{18}$ In neonatal candidiasis with CNS involvement, it is strongly recommended by IDSA to remove all CNS devices (including ventriculostomy drains and shunts), if possible. ${ }^{31}$

\section{Conclusion}

Neonates with LBW (<2500 g) and early gestational age (29-32 weeks) are at increased risk of developing invasive candidiasis. Other risk factors include vaginal birth, central catheters, day of life (>7), use of broad spectrum antibiotics, antenatal steroids, premature rupture of membranes, mechanical ventilation, necrotizing enterocolitis and parenteral nutrition. High mortality rate associated with invasive candidiasis in neonates necessitates the need to fill the gaps in its diagnosis and treatment. Also, adverse neurodevelopmental outcomes have been reported in neonates with invasive candidiasis. Use of empiric anti-fungal therapy is suggested to help in improving neurodevelopmental outcomes in neonates with invasive candidiasis. Culturing of blood and other body fluids is the conventional method used for diagnosis of invasive candidiasis. However, it leads to delay in diagnosis due to time-consuming procedure. Other molecular techniques including PCR and biomarkers are being investigated to be used for rapid diagnosis; however, no recommendations have been made in international guidelines. Also, there is lack of substantial data on efficacy and safety of antifungals in infants with invasive candidiasis. However, recommendations have been made in guidelines based on the available data. The guidelines have strongly recommended the use of D-Amp B and flucanozole in neonatal candidiasis. Other therapies including liposomal formulations of Amp B, oral nystatin, echinocandins, flucytosine and Lactoferrin are suggested as alternatives. In future, there is a need to conduct more studies in large population size and assess the long-term outcomes following antifungal prophylaxis in neonatal invasive candidiasis.

\section{Acknowledgments}

None.

\section{Conflicts of interest}

The authors declare no conflicts of interest.

\section{References}

1. Ben Abdeljelil J, Saghrouni F, Nouri S, et al. Neonatal invasive candidiasis in Tunisian hospital:incidence, risk factors, distribution of species and antifungal susceptibility. Mycoses. 2012;55(6):493-500. 
2. Greenberg RG, Benjamin DK, Gantz MG, et al. Empiric antifungal therapy and outcomes in extremely low birth weight infants with invasive candidiasis. J Pediatr. 2012;161(2):264-269.

3. Xia H, Wu H, Xia S, et al. Invasive Candidiasis in preterm neonates in China: a retrospective study from 11 NICUS during 2009-2011. Pediatr Infect Dis J. 2014;33(1):106-109.

4. Sriram B, Agarwal PK, Tee NW, et al. Systemic Candidiasis in Extremely Low Birthweight (ELBW) Neonates Despite the Routine Use of Topical Miconazole Prophylaxis: Trends, Risk Factors and Outcomes over an 11-Year Period. Ann Acad Med Singapore. 2014;43(5):255-262.

5. Pammi M, Holland L, Butler G, et al. Candida parapsilosis is a significant neonatal pathogen:a systematic review and meta-analysis. Pediatr Infect Dis J. 2013;32(5):e206-e216.

6. Aruna C, Seetharam K. congenital candidiasis. Indian Dermatol Online J. 2014;5(Suppl 1):S44-547.

7. Diana A, Martin G, Ecoffey M, et al. Congenital cutaneous candidiasis $(\mathrm{CCC})$ : a rare skin disorder of the neonate. Swiss society of neonatology.

8. Kelly MS, Benjamin DK, Smith PB. The epidemiology and diagnosis of invasive candidiasis among premature infants. Clin Perinatol. 2015;42(1):105-117.

9. Pan N, Herzog R, Blanco JS, et al. Candida albicans osteomyelitis in an infant:a case report and literature review. J Pediatr Orthop B. 2013;22(5):491-497.

10. Fanaroff, Martin's Neonatal-Perinatal Medicine: Diseases of the Fetus and Infant. 10th edn, In: Martin RJ FA, Walsh FC, editors, Elsevier, Amsterdam, Netherlands.

11. Aliaga S, Clark RH, Laughon M, et al. Changes in the incidence of candidiasis in neonatal intensive care units. Pediatrics. 2014;133(2):236242 .

12. Chitnis AS, Magill SS, Edwards JR, et al. Trends in Candida central line-associated bloodstream infections among NICUs, 1999-2009. Pediatrics. 2012;130(1):e46-e52.

13. Barton M, O’Brien K, Robinson JL, et al. Invasive candidiasis in low birth weight preterm infants:risk factors, clinical course and outcome in a prospective multicenter study of cases and their matched controls. BMC Infect Dis. 2014;14:327.

14. Greenberg RG, Benjamin DK. Neonatal candidiasis:diagnosis, prevention, and treatment. The Journal of infection. 2014;69:S19-S22.

15. Lestner JM, Smith PB, Cohen-Wolkowiez M, et al. Antifungal agents and therapy for infants and children with invasive fungal infections:a pharmacological perspective. Br J Clin Pharmacol. 2013;75(6):13811395 .

16. Trofa D, Gacser A, Nosanchuk JD. Candida parapsilosis, an emerging fungal pathogen. Clin Microbiol Rev. 2008;21(4):606-625.

17. Khan EA, Choudhry S, Fatima M, et al. Clinical spectrum, management and outcome of neonatal candidiasis. J Pak Med Assoc. 2015;65(11):1206-1209.

18. Benjamin DK, Stoll BJ, Fanaroff AA, et al. Neonatal candidiasis among extremely low birth weight infants:risk factors, mortality rates, and neurodevelopmental outcomes at 18 to 22 months. Pediatrics. 2006;117(1):84-92.

19. de Haan TR, Beckers L, de Jonge RC, et al. Neonatal gram negative and Candida sepsis survival and neurodevelopmental outcome at the corrected age of 24 months. PloS One. 2013;8(3):e59214.

20. Adams-Chapman I, Bann CM, Das A, et al. Neurodevelopmental outcome of extremely low birth weight infants with Candida infection. $J$ Pediatr. 2013;163(4):961-967.

21. Downey LC, Smith PB, Benjamin DK, et al. Recent Advances in the Detection of Neonatal Candidiasis. Curr Fungal Infect Rep. 2010;4(1):17-22.

22. Cohen-Wolkowiez M, Smith PB, Mangum B, et al. Neonatal Candida meningitis: significance of cerebrospinal fluid parameters and blood cultures. J Perinatol. 2007;27(2):97-100.

23. Radic M, Goic-Barisic I, Novak A, et al. Evaluation of PNA FISH(R) Yeast Traffic Light in identification of Candida species from blood and non-blood culture specimens. Medical mycology. 2016;54(6):654-658.

24. Kasper DC, Altiok I, Mechtler TP, et al. Molecular detection of late-onset neonatal sepsis in premature infants using small blood volumes:proofof-concept. Neonatology. 2013;103(4):268-273.

25. Taira CL, Okay TS, Delgado AF, et al. A multiplex nested PCR for the detection and identification of Candida species in blood samples of critically ill paediatric patients. BMC infectious diseases. 2014;14:406.

26. Pammi M, Flores A, Leeflang M, et al. Molecular assays in the diagnosis of neonatal sepsis:a systematic review and meta-analysis. Pediatrics. 2011;128(4):e973-985.

27. Paolucci M, Landini MP, Sambri V. How can the microbiologist help in diagnosing neonatal sepsis?. International journal of pediatrics. 2012;2012:12.

28. Tragiannidis A, Tsoulas C, Groll AH. Invasive candidiasis and candidaemia in neonates and children:update on current guidelines. Mycoses. 2015;58(1):10-21.

29. Zhao D, Qiu G, Luo Z, et al. Platelet parameters and (1,3)-beta-Dglucan as a diagnostic and prognostic marker of invasive fungal disease in preterm infants. PloS one. 2015;10(4):e0123907.

30. Hope WW, Castagnola E, Groll AH, et al. ESCMID* guideline for the diagnosis and management of Candida diseases 2012:prevention and management of invasive infections in neonates and children caused by Candida spp. Clinical microbiology and infection :the official publication of the European Society of Clinical Microbiology and Infectious Diseases. 2012;18(7):38-52.

31. Pappas PG, Kauffman CA, Andes DR, et al. Clinical Practice Guideline for the Management of Candidiasis:2016 Update by the Infectious Diseases Society of America. Clin Infect Dis. 2016;62(4):e1-50.

32. Holler B, Omar SA, Farid MD, et al. Effects of fluid and electrolyte management on amphotericin B-induced nephrotoxicity among extremely low birth weight infants. Pediatrics. 2004;113(6):e608-616.

33. Cetin H, Yalaz M, Akisu M, et al. The efficacy of two different lipidbased amphotericin B in neonatal Candida septicemia. Pediatrics international: official journal of the Japan Pediatric Society. 2005;47(6):676-680.

34. Walsh TJ, Seibel NL, Arndt C, et al. Amphotericin B lipid complex in pediatric patients with invasive fungal infections. The Pediatric infectious disease journal. 1999;18(8):702-708.

35. Ascher SB, Smith PB, Watt K, et al. Antifungal therapy and outcomes in infants with invasive Candida infections. The Pediatric infectious disease journal. 2012;31(5):439-443.

36. Benjamin DK, Hudak ML, Duara S, et al. Effect of fluconazole prophylaxis on candidiasis and mortality in premature infants:a randomized clinical trial. Jama. 2014;311(17):1742-1749.

37. Lee J, Kim HS, Shin SH, et al. Efficacy and safety of fluconazole prophylaxis in extremely low birth weight infants:multicenter pre-post cohort study. BMC pediatrics. 2016;16:67.

38. Tripathi N, Watt K, Benjamin DK. Treatment and prophylaxis of invasive candidiasis. Seminars in perinatology. 2012;36(6):416-423.

39. Arrieta AC, Maddison P, Groll AH. Safety of micafungin in pediatric clinical trials. The Pediatric infectious disease journal. 2011;30(6):e97e102. 
40. Benjamin DK, Smith PB, Arrieta A, et al. Safety and pharmacokinetics of repeat-dose micafungin in young infants. Clinical pharmacology and therapeutics. 2010;87(1):93-99.

41. Manzoni $\mathrm{P}, \mathrm{Wu} \mathrm{C}$, Tweddle $\mathrm{L}$, et al. Micafungin in premature and nonpremature infants:a systematic review of 9 clinical trials. The Pediatric infectious disease journal. 2014;33(11):e291-298.

42. Mohamed WA, Ismail M. A randomized, double-blind, prospective study of caspofungin vs. amphotericin B for the treatment of invasive candidiasis in newborn infants. Journal of tropical pediatrics. 2012;58(1):25-30.

43. Jeon GW, Sin JB. Successful caspofungin treatment of persistent candidemia in extreme prematurity at 23 and 24 weeks' gestation. Journal of the Formosan Medical Association Taiwan yi zhi. 2014;113(3):191-194.

44. Al-Sweih N, Ahmad S, Joseph L, et al. Candida fermentation as a cause of persistent fungemia in a preterm neonate successfully treated by combination therapy with amphotericin B and caspofungin. Journal of clinical microbiology. 2015;53(3):1038-1041.

45. Turan O, Ergenekon E, Hirfanoglu IM, et al. Combination antifungal therapy with voriconazole for persistent candidemia in very low birth weight neonates. The Turkish journal of pediatrics. 2011;53(1):19-26.

46. Botero-Calderon L, Benjamin DK, Cohen-Wolkowiez M. Advances in the treatment of invasive neonatal candidiasis. Expert opinion on pharmacotherapy. 2015;16(7):1035-1048

47. Rao SC, Athalye-Jape GK, Deshpande GC, et al. Probiotic Supplementation and Late-Onset Sepsis in Preterm Infants:A Metaanalysis. Pediatrics. 2016;137(3):e20153684.

48. Zhang GQ, Hu HJ, Liu CY, et al. Probiotics for Preventing LateOnset Sepsis in Preterm Neonates:A PRISMA-Compliant Systematic Review and Meta-Analysis of Randomized Controlled Trials. Medicine. 2016;95(8):e2581
49. Kumar S, Singhi S. Role of Probiotics in prevention of Candida colonization and invasive andidiasis. The journal of maternal-fetal \& neonatal medicine :the official journal of the European Association of Perinatal Medicine, the Federation of Asia and Oceania Perinatal Societies, the International Society of Perinatal Obstet. 2016;29(5):818819.

50. Peter G Pappas, Carol A Kauffman, David R Andes, et al. Clinical Practice Guideline for the Management of Candidiasis:2016 Update by the Infectious Diseases Society of America. Clin Infect Dis. 2016;62(4):e1-50.

51. Lee JH, Hornik CP, Benjamin DK, et al. Risk Factors for Invasive Candidiasis in Infants $>1500 \mathrm{~g}$ Birth Weight. Pediatr Infect Dis J. 2013;32(3):222-226

52. Oeser C, Vergnano S, Naidoo R, et al. Neonatal invasive fungal infection in England 2004-2010. Clinical Microbiology and Infection. 2014;20(9):936-941.

53. Wadile RG, Bhate VM. Study of clinical spectrum and risk factors of neonatal candidemia. Indian J Pathol Microbiol. 2015;58(4):472-474.

54. Goudjil S, Kongolo G, Dusol L, et al. (1-3)- $\beta-D-$ glucan levels in candidiasis infections in the critically ill neonate. J Matern Fetal Neonatal Med. 2013;26(1):44-48.

55. Oliveri S, Trovato L, Betta P, et al. Experience with the Platelia Candida ELISA for the diagnosis of invasive candidosis in neonatal patients. Clinical Microbiology and Infection. 2008;14(4):391-393.

56. Mularoni A, Furfaro E, Faraci M, et al. High Levels of beta-D-glucan in immunocompromised children with proven invasive fungal disease. Clin Vaccine Immunol. 2010;17(5):882-883. 